\title{
Long-term efficacy and tolerability of intranasal fentanyl in the treatment of breakthrough cancer pain
}

\author{
Sebastiano Mercadante • Renato Vellucci • \\ Arturo Cuomo • Claudio Adile • Andrea Cortegiani • \\ Alessandro Valle • Patrizia Villari • Alessandra Casuccio
}

Received: 8 August 2014 / Accepted: 17 October 2014 / Published online: 29 October 2014

(C) Springer-Verlag Berlin Heidelberg 2014

\begin{abstract}
Purpose The aim of the present study was to assess the longterm tolerability and efficacy of intranasal fentanyl (INFS) in opioid-tolerant patients with breakthrough cancer pain (BTP). Patients and methods A 6 months, observational, prospective, cohort study design was employed to follow advanced cancer patients with BTP receiving INFS under routine clinical practice. Eligible adult cancer patients suffering from BTP had been prescribed INFS at effective doses. Data were collected at $\mathrm{T} 0$ and at month intervals for six months. The principal outcomes were the evaluation of possible serious adverse effects with prolonged use of INFS, the efficacy of BTP treatment with INFS, the quality of sleep, the rate of INFS discontinuation, and reasons for that.
\end{abstract}

\footnotetext{
S. Mercadante $(\bowtie) \cdot$ C. Adile $\cdot$ P. Villari

Pain Relief and Palliative Care Unit, La Maddalena Cancer Center, Via San Lorenzo 312, 90146 Palermo, Italy

e-mail: terapiadeldolore@lamaddalenanet.it

R. Vellucci

Pain Unit, Ospedale, Careggi, Florence, Italy

A. Cuomo

Anesthesia and Intensive Care, Pain Unit, National Cancer Institute Pascale, Naples, Italy

\section{A. Cortegiani}

Department of Biopathology, Medical and Forensic Biotechnologies (DIBIMEF), Section of Anesthesia, Analgesia, Intensive Care and Emergency, Policlinico "P. Giaccone", University of Palermo, Palermo, Italy

A. Valle

FARO Foundation, Turin, Italy

A. Casuccio

Department of Sciences for Health Promotion and Mother-Child Care "G. D'Alessandro", University of Palermo, Palermo, Italy
}

Results Seventy-five patients were surveyed. Thirty-four patients $(45.3 \%)$ had a follow-up at 3 months, and twelve patients $(16 \%)$ were followed up at 6 months. The mean opioid doses, expressed as oral morphine equivalents, ranged 111-180 mg/day, while the mean INFS doses were 87$119 \mu \mathrm{g}$. Adverse effects were reported in a minority of patients and were considered to be associated with opioid therapy used for background pain. The quality of sleep significantly improved during the first 3-4 months. Finally, efficacy based on a general impression regarding the efficacy of INFS was good-excellent in most patients and statistically improved in time up to the third month.

Conclusion The long-term use of INFS in advanced cancer patients is effective and safe. No serious adverse effects were found up to six months of assessment. The level of quality of sleep and patients' satisfaction was relatively good, considering the advanced stage of disease.

Keywords Cancer pain · Breakthrough pain · Intranasal fentanyl $\cdot$ Tolerability

\section{Introduction}

Breakthrough cancer pain (BTP) is defined as a transient exacerbation of pain that occurs on a background of otherwise stable persistent pain [1]. BTP affects more than half of all patients with cancer and has a substantial impact on quality of life, including detrimental effects on activities of daily living, sleep, social relationships, and enjoyment of life [2]. Consequently, control of BTP represents an important therapeutic goal in the care of patients with cancer. Typical episodes of BTP are characterized by a rapid onset, short duration, and severe intensity [3]. As a result, current treatments for BTP primarily involve immediate-release opioids, such as morphine, oxycodone, and hydromorphone. However, the 
hydrophilic nature of such opioids means that they are primarily absorbed through the gut, leaving them prone to firstpass metabolism and slow onset of action. Thus, these drugs can take about 30 min to produce an analgesic effect, which then lasts for about $4 \mathrm{~h}$. This approach may be suitable for treating some pain conditions with a slow onset or administering such drugs before an expected predictable pain event. Indeed, oral immediate-release opioids do not reach peak activity until 30-45 min after administration [4] and are therefore unable to match the time-pain profile of BTP episodes. The majority of BTP episodes require a treatment with a fast onset of action and short duration of effect. Fentanyl is a highly lipophilic opioid and is therefore well-suited to oral transmucosal administration.

A range of fast-acting fentanyl formulations has been developed and are approved with these purposes. These agents allow rapid absorption and quick onset of effect, with good levels of acceptability to patients [5]. In some conditions, oral mucosa may be damaged and drug absorption may be problematic. A possible alternative approach to the treatment of BTP is to take advantage of the favorable absorption and characteristics of the nasal mucosa, which allows for rapid drug absorption and, hence, rapid onset of analgesia [6].

Intranasal fentanyl (INFS) is a treatment option approved for the management of BTP in adult cancer patients already receiving maintenance opioid therapy for chronic cancer pain. INFS has been used in various settings, populations, and different circumstances [6-36]. Clinical studies in cancer patients have shown that INFS provides clinically relevant reductions in pain intensity [37], with superior efficacy and patient preference, compared to oral transmucosal fentanyl citrate (OTFC) [38]. Previous evaluations of INFS were conducted through controlled clinical trials. From a systematic literature review, INFS is expected to provide the greatest improvement in the treatment of BTP [39]. The aim of the present study was to assess the long-term tolerability and efficacy of INFS in opioid-tolerant patients with BTP.

\section{Patients and methods}

A non-interventional, 6 months, observational, prospective, cohort study design was employed to follow advanced cancer patients with BTP receiving INFS under routine clinical practice. The study was conducted according to the Declaration of Helsinki and Ethics Committees granted ethical approval. Study participation had no impact on the patient (except for the collection of informed consent, requested after the treatment decision); the decision to prescribe INFS had been determined as part of current practice. Patients were not subjected to additional procedures; participating study physicians were responsible for all decisions regarding patient care.
Eligible patients were adult cancer patients (home care or outpatients) suffering from BTP who had been prescribed INFS at effective doses. All patients provided informed consent for the collection of data prior to study inclusion. Adult cancer patients (aged $\geq 18$ years) with stable cancer related pain were recruited into the study. A convenience sample of patients using INFS for three consecutive days effectively was surveyed for a follow-up of six months.

Inclusion criteria were patients with receiving opioids for background pain and having episodes of BTP treated with INFS, a well-controlled background pain while receiving a fixed-schedule oral opioid regimen of $\geq 60$ oral morphine equivalents of different opioids (less than $4 / 10$ of pain intensity on a numerical scale from 0 to 10$), 1-3$ episodes of BTP per day, and an expected survival of greater than 6 months

Exclusion criteria included uncontrolled or rapidly escalating pain, any clinically significant condition that would, in the investigator's opinion, precluded study participation or increase the risk of administering potent opioids, the use of monoamine oxidase inhibitors within 14 days, or other anticancer interventions with a potential to interfere with the treatment, patients severely ill.

Patients were recruited from four study centers across Italy. Data were collected at $\mathrm{T} 0$ and at month intervals for six months (T1 to T6). In case of need, visits or phone contacts were anticipated. Data were mainly obtained from source data, by the investigator, and were collected for INFS-treated patients only. The trend of INFS doses in time was also recorded. Adverse reactions were recorded, with an intensity graded from 0 to three (absence, mild, moderate, and severe). Efficacy was based on a general impression regarding the efficacy of INFS with the following scale: 1 (poor), 2 (acceptable), 3 (good), 4 (very good), 5 (excellent). [42]. Quality of sleep was rated with the following scale: 0 (disturbed), 1 (frequent arousals), 2 (good), 3 (very good).

The principal outcomes were the evaluation of possible serious adverse effects with prolonged use of INFS, the efficacy of BTP treatment with INFS, the quality of sleep, the rate of INFS discontinuation, and reasons for that. Secondary outcomes were the relationship with the dose of INFS and opioid doses used for background analgesia commonly changed to maintain an acceptable background pain $(\leq 4 / 10$ on a numerical scale $0-10$ ).

\section{Statistical analysis}

Data were analyzed by the IBM SPSS Software 21.0 version (SPSS, Inc., Chicago, Ill, US) and the Epi Info software, version 3.2.2, (Centers for Disease Control and Prevention). Statistical analysis of quantitative and qualitative data, included descriptive statistics, was performed for all the items. Frequency analysis was performed with chi-square test. The paired Wilcoxon signed-rank test and ANOVA test for 
Table 1 Patients' demographics and baseline characteristics

\begin{tabular}{ll} 
Mean age, years (SD) & $64.8(12.7)$ \\
Male, $n(\%)$ & $65(86.7)$ \\
Karnofsky & $69.9(26.3)$ \\
Site of primary tumor, $n(\%)$ & $n(\%)$ \\
Lung/respiratory & $16(21.3)$ \\
Colon/rectum & $13(17.3)$ \\
Breast & $18(24.0)$ \\
Head and neck & $6(8.1)$ \\
Urogenital & $13(17.3)$ \\
Pancreas & $1(1.3)$ \\
Liver & $3(4)$ \\
Others & $5(6.7)$ \\
\hline
\end{tabular}

repeated measures were used to compare non-parametric and parametric variables, respectively, at different intervals. Pearson's correlation test was used to test for a comparison between opioids doses, expressed as oral morphine equivalents (OME) and doses of INFS. All $P$ values were two-sided, and $P$ values less than 0.05 were considered to indicate statistical significance.

\section{Results}

Seventy-five patients were surveyed. Thirty-four patients (45.3\%) had a follow-up at 3 months, and twelve patients $(16 \%)$ were followed up at 6 months. The characteristics of patients are described in Table 1. Seven patients dropped out at T1: one patient died and six were unavailable. Eleven patients dropped out at T2: six patients died, one was unavailable, and four were lost in the follow-up. Twenty-three patients dropped out at T3: 17 were unavailable and six were lost in the follow-up. Seven patients dropped out at T4: two patients died and five patients were unavailable. Eight patients dropped out at T5: six died and two were unavailable. Finally, seven patients dropped out at T6: two patients died, two were unavailable, and three were lost in the follow-up. No patient discontinued the treatment for severe adverse effects, and no aberrant drug-related behavior was observed.

Opioids and doses, expressed as oral morphine equivalents (OME) given for background analgesia, and doses of INFS are reported in Table 2. Mean opioid doses ranged 111$180 \mathrm{mg}$ /day, while the mean INFS doses were 87-119 $\mu \mathrm{g}$. Doses of INFS and OMR significantly increased in time, unless some certain time intervals, possibly due to a lower number of patients continuing the study at months 5 and 6 . There was a statistical correlation between INFS and OME doses at $\mathrm{T} 0$ and $\mathrm{T} 1$ (Pearson correlation coefficient 0.545, $P<0.0005$, and $0.248, P=0.043$, respectively), but not at the subsequent time intervals.

Adverse effects were reported in a minority of patients and were considered to be associated with opioid therapy used for background pain. The intensity was mild and only few patients presented symptoms of moderate intensity (Table 3 ).

The quality of sleep significantly improved during the first 3-4 months (Table 4). Finally, efficacy based on a general impression regarding the efficacy of INFS was good-excellent in most patients and statistically improved in time up to the third month (Table 5).

\section{Discussion}

This long-term study in advanced cancer patients with BTP has shown that INFS was well tolerated for prolonged periods of time and was effective in controlling episodes of BTP, according to the general impression reported by patients. Adverse effects were of mild-moderate intensity and mostly attributed to opioids administered for background pain. Quality of sleep was relatively maintained in time, and no

Table 2 Number of patients using the different opioids, mean oral morphine equivalents (OME) (SD), and mean INFS dose (SD) at the different time intervals

\begin{tabular}{|c|c|c|c|c|c|c|c|}
\hline & $\mathrm{T} 0$ & $\mathrm{~T} 1$ & $\mathrm{~T} 2$ & $\mathrm{~T} 3$ & $\mathrm{~T} 4$ & $\mathrm{~T} 5$ & T6 \\
\hline $\mathrm{N}^{\circ}$ pts & 75 & 68 & 57 & 34 & 27 & 19 & 12 \\
\hline TD buprenorphine & 5 & 3 & 1 & 1 & 1 & - & - \\
\hline TD Fentanyl & 18 & 17 & 14 & 8 & 8 & 8 & 5 \\
\hline Morphine & 12 & 12 & 9 & 7 & 4 & 3 & 2 \\
\hline Hydromorphone & 6 & 3 & 1 & - & - & - & - \\
\hline Oxycodone & 28 & 25 & 23 & 16 & 12 & 7 & 4 \\
\hline Tapentadol & 5 & 5 & 5 & - & - & - & - \\
\hline Methadone & 2 & 1 & 1 & 1 & 1 & 1 & 1 \\
\hline OME (mg) & $121(106)$ & $122(92)$ & $138(106)^{*}$ & $111(105)$ & $138(112)^{*}$ & $142(132)$ & $180(181)$ \\
\hline INFS $(\mu \mathrm{g})$ & $87(70)$ & $90(49)^{*}$ & $113(63)^{*}$ & $103(75)^{*}$ & $108(82)$ & $119(94)$ & $100(59)$ \\
\hline
\end{tabular}

$* P<0.05$ versus $\mathrm{T} 0$ 
Table 3 Number of patients (\%) presenting adverse effects of mild-moderate intensity at different time intervals (above) and number of patients (below) presenting specific adverse effects with a moderate intensity

\begin{tabular}{|c|c|c|c|c|c|c|c|}
\hline & T0 & $\mathrm{T} 1$ & $\mathrm{~T} 2$ & $\mathrm{~T} 3$ & $\mathrm{~T} 4$ & $\mathrm{~T} 5$ & T6 \\
\hline $\mathrm{N}^{\circ}$ pts & 75 & 68 & 57 & 34 & 27 & 19 & 12 \\
\hline Adverse effects, $n(\%)$ & $21(28)$ & $28(41)$ & $24(42)$ & $14(41)$ & $11(41)$ & $7(37)$ & $3(25)$ \\
\hline \multicolumn{8}{|c|}{ Symptoms with moderate intensity } \\
\hline All & $2(2.7)$ & $15(22)$ & $3(5.3)$ & $1(2.9)$ & $3(11.1)$ & $1(5.2)$ & $0(0.0)$ \\
\hline Headache & & & $1(1.4)$ & $1(1.7)$ & & & \\
\hline Drowsiness & & $1(1.3)$ & $11(16)$ & & & & \\
\hline Constipation & & $1(1.3)$ & $2(2.9)$ & $2(3.5)$ & $1(2.9)$ & $2(7.4)$ & $1(5.2)$ \\
\hline Dizziness & & & $1(1.4)$ & & & $1(3.7)$ & \\
\hline
\end{tabular}

discontinuation of treatment was due to INFS-related adverse effects. Rather, drop-out rate was relatively expected, due to the nature of the study assessing advanced cancer patients with a limited survival. Finally, no aberrant drug-related behavior was observed.

Many cancer patients are managed for prolonged periods of time and treatment of BTP may last several months. Therefore, it is of paramount importance to gather information about the long-term use of medications commonly used for BTP. Data regarding long-term use of second generation fentanyl products in cancer patients are limited. A series of extension studies of previous controlled studies have shown that fentanyl products are well tolerated and effective. Fentanyl buccal tablet (FBT) was well tolerated and had a favorable safety profile in a long-term (up to 12 months) management of BTP. Adverse effects were unrelated to study drug [40]. Subsequently, FBT has been assessed for 12 weeks in an open-label extension phase after completing a controlled study with oral opioids in both cancer and non-cancer patients. Most adverse effects were attributed to opioids given for background pain or were of minor intensity. Only one patient discontinued the treatment, due to a withdrawal syndrome after stopping to take opioids. A better functional improvement and satisfaction with FBT vs short acting opioids were reported [41]. Sublingual fentanyl (SLF) was assessed in cancer patients in a long-term safety phase of 12 months for the management of BTP. Only one patient dropped out for a serious adverse effect considered as possibly related to study medication [42].

Table 4 Levels of quality of sleep at the different intervals

\begin{tabular}{lllllllll}
\hline & & $\mathrm{T} 0$ & $\mathrm{~T} 1$ & $\mathrm{~T} 2$ & $\mathrm{~T} 3$ & $\mathrm{~T} 4$ & $\mathrm{~T} 5$ & $\mathrm{~T} 6$ \\
\hline $\mathrm{N}^{\circ}$ pts & & 75 & 68 & 57 & 34 & 27 & 19 & 12 \\
Sleep scores & 1 & 2 & 12 & 7 & 2 & 3 & 3 & 2 \\
& 2 & 16 & 28 & 26 & 9 & 5 & 4 & 5 \\
& 3 & 25 & 28 & 24 & 23 & 19 & 12 & 5 \\
& 4 & 32 & - & - & - & - & - & - \\
$P$ & & & .0005 & .0005 & .0005 & .043 & .103 & .287
\end{tabular}

The long-term tolerability, efficacy, and acceptability of fentanyl pectin spray (FPNS) for BTP has been recently reported in a 16 weeks period. FPNS was generally well tolerated and accepted by patients, and adverse effects were of mild to moderate intensity. One third of patients completed 16 weeks of treatment. Nine patients discontinued for adverse effects due to the study drug. Severe local damage was reported in one patient [43]. In a subsequent extension study, the need of discontinuation of FPNS was not related to the drug, and only one patient who misused the drug had serious adverse effects [44]. One study assessed INFS for three months. One-third of patients were still receiving INFS at the end of the study. Serious adverse effects were reported in about $3 \%$ of patients who discontinued INFS. Other than death, discontinuation occurred for lack of efficacy or other reasons. The treatment improved patients' satisfaction and brief pain inventory from baseline to the fourth week [45].

Of interest, in almost these studies, the effective dose was achieved after an open-label phase of dose titration of about two weeks, although most patients were administered the lowest strengths. After performing a controlled phase, they entered an extension phase. The majority of patients maintained the doses to which they were titrated throughout their participation in the study. When available, the doses of opioids used for background pain was unchanged.

Differently from the findings reported in these studies, in the present long-term study of INFS, physicians could use either dose titration or doses proportional to the opioid basal

Table 5 Distribution of patients according to the INFS efficacy

\begin{tabular}{lllllllll}
\hline & & T0 & T1 & T2 & T3 & T4 & T5 & T6 \\
\hline $\mathrm{N}^{\circ}$ pts & & 75 & 68 & 57 & 34 & 27 & 19 & 12 \\
Efficacy score & 1 & - & - & 1 & 1 & - & - & - \\
& 2 & 11 & 5 & 2 & - & - & 2 & - \\
& 3 & 14 & 16 & 15 & 2 & - & 2 & 2 \\
& 4 & 27 & 25 & 19 & 15 & 9 & 7 & 5 \\
& 5 & 23 & 22 & 20 & 16 & 18 & 8 & 5 \\
& $P$ & & .0005 & .0005 & .063 & .357 & .440 & .766 \\
\hline
\end{tabular}


regimen for dosing INFS and doses were then changed according to the clinical need. Increases in time of both opioid doses for background pain and INFS doses were limited. A dose correlation was found for the first month, and disappeared at the subsequent time intervals, possibly because of the decrease in the number of patients who were continuing the study. On average, there was a proportion between the mean OME and INFS doses (111-180 mg/day, and 87$119 \mu \mathrm{g}$, respectively). It has been shown that INFS and FPNS in doses proportional to basal opioid regimen are equally safe and effective for the management of breakthrough pain in cancer patients. These data provide new insights on the use of nasal preparations of fentanyl [46].

The treatment was effective and able to maintain sleep quality at acceptable levels, rather than being particularly accepted by patients who were particularly satisfied with the treatment. Most of patients discontinued the treatment for death or unavailability to continue study participation, and about half of patients dropped out from the study. This finding was expected and reflects the underlying disease states of patients and the long duration of the trial. No patient discontinued the study drug for serious adverse effects, which were mild-moderate in intensity and attributed to background opioids or the underlying disease.

There are limitations to consider, which are inherent with studies in advanced cancer patients. Patient numbers were low after some weeks, due to a physiological drop-out percentage, typical of a cohort of patients with progressive disease or unavailability of patients to maintain the contact with the study center. No comparator arm was used. However, this approach was deemed to not have contributed to the objective of the description of the study, which regarded the safety and efficacy of INFS in a real clinical world. The treatment was changed according to the clinical need, without restricted obligations of a protocol. Indeed, this reflects the real life of advanced cancer patients.

In conclusion, this long-term study has shown that the use of INFS in advanced cancer patients is effective and safe. No serious adverse effects were found up to six months of assessment. Doses of opioids used for background analgesia and INFS doses may be changed according to clinical needs, as expected in advanced cancer patients. The level of quality of sleep and patients' satisfaction was relatively good, considering the advanced stage of disease, although a multitude of factors may have an influence. Other studies on large scale should confirm the safety and efficacy of fentanyl products for the management of BTP.

Conflict of interest Prof. Mercadante is a consultant for the following companies: Molteni, Grunenthal, Janssen, Mundipharma, and Takeda.

Authors had full control of all primary data and agree to allow the journal to review data if requested.

\section{References}

1. Portenoy RK, Hagen NA (1990) Breakthrough pain: definition, prevalence and characteristics. Pain 41:273-81

2. Portenoy RK, Payne D, Jacobsen P (1999) Breakthrough pain: characteristics and impact in patients with cancer pain. Pain 81:129-34

3. Mercadante S, Arcuri E (1998) Breakthrough pain in cancer patients: pathophysiology and treatment. Cancer Treat Rev 24:425-32

4. Zeppetella G (2009) Dynamics of breakthrough pain vs pharmacokinetics of oral morphine: implications for management. Eur J Cancer Care 18:331-7

5. Mercadante S (2012) Pharmacotherapy for breakthrough cancer pain. Drugs 72:181-90

6. Grassin-Delyle S, Buenestado A, Naline E et al (2012) Intranasal drug delivery: an efficient and non-invasive route for systemic administration: focus on opioids. Pharmacol Ther 134:366-79

7. Thronæs M, Kaasa S, Dale O (2014) A pilot study of nasal fentanyl for patient controlled treatment of cancer pain. J Opioid Manag 10: 21-8

8. Karlsen AP, Pedersen DM, Trautner S, Dahl JB, Hansen MS (2014) Safety of intranasal fentanyl in the out-of-hospital setting: a prospective observational study. Ann Emerg Med 63:699-703

9. Nave R, Schmitt H, Popper L (2013) Faster absorption and higher systemic bioavailability of intranasal fentanyl spray compared to oral transmucosal fentanyl citrate in healthy subjects. Drug Deliv 20:21623

10. Plock N, Facius A, Hartmann L, Baumann S, Nave R (2013) An innovative phase I population pharmacokinetic approach to investigate the pharmacokinetics of an intranasal fentanyl spray in healthy subjects. Int J Clin Pharmacol Ther 51:495-508

11. Smith HS (2013) Considerations in selecting rapid-onset opioids for the management of breakthrough pain. J Pain Res 6:189-200

12. Lötsch J, Walter C, Parnham MJ, Oertel BG, Geisslinger G (2013) Pharmacokinetics of non-intravenous formulations of fentanyl. Clin Pharmacokinet 52:23-36

13. Harlos MS, Stenekes S, Lambert D, Hohl C, Chochinov HM (2013) Intranasal fentanyl in the palliative care of newborns and infants. J Pain Symptom Manag 46:265-74

14. Dietrich E, Gums JG (2012) Intranasal fentanyl spray: a novel dosage form for the treatment of breakthrough cancer pain. Ann Pharmacother 46:1382-91

15. Nave R, Connolly SM, Popper L, Lahu G, Schmitt H (2012) Singledose and multi-dose delivery systems for intranasal fentanyl spray are bioequivalent as demonstrated in a replicate pharmacokinetic study. Int J Clin Pharmacol Ther 50:751-9

16. Kaessner N, Nave R, Roepcke S, Facius A, Lahu G (2012) Population pharmacokinetic meta-analysis of intranasal fentanyl spray as a means to enrich pharmacokinetic information for patients with cancer breakthrough pain. Int J Clin Pharmacol Ther 50:665-77

17. Veldhorst-Janssen NM, Fiddelers AA, Zandstra H et al (2012) Patient satisfaction with intranasal fentanyl for breakthrough pain. J Palliat Med 15:631-2

18. Barrett MJ, Cronin J, Murphy A et al (2012) Intranasal fentanyl versus intravenous morphine in the emergency department treatment of severe painful sickle cell crises in children: study protocol for a randomised controlled trial. Trials 13:74

19. Hansen MS, Mathiesen O, Trautner S, Dahl JB (2012) Intranasal fentanyl in the treatment of acute pain - a systematic review. Acta Anaesthesiol Scand 56:407-19

20. Kusre SR (2011) Towards evidence based emergency medicine: best BETs from the Manchester Royal Infirmary. Bet 4: is intranasal fentanyl better than parenteral morphine for managing acute severe pain in children? Emerg Med J 28:1077-8

21. Mudd S (2011) Intranasal fentanyl for pain management in children: a systematic review of the literature. J Pediatr Health Care 25:316-22 
22. Mystakidou K, Panagiotou I, Gouliamos A (2011) Fentanyl nasal spray for the treatment of cancer pain. Expert Opin Pharmacother 12: 1653-9

23. Prommer E, Thompson L (2011) Intranasal fentanyl for pain control: current status with a focus on patient considerations. Patient Prefer Adherence 5:157-64

24. Borland M, Milsom S, Esson A (2011) Equivalency of two concentrations of fentanyl administered by the intranasal route for acute analgesia in children in a paediatric emergency department: a randomized controlled trial. Emerg Med Australas 23:202-8

25. Vissers DC, Lenre M, Tolley K et al (2011) An economic evaluation of short-acting opioids for treatment of breakthrough pain in patients with cancer. Value Health 14:274-8

26. Leppert W (2010) Role of intranasal fentanyl in breakthrough pain management in cancer patients. Cancer Manag Res 2:225-32

27. Saunders M, Adelgais K, Nelson D (2010) Use of intranasal fentanyl for the relief of pediatric orthopedic trauma pain. Acad Emerg Med 17:1155-61

28. Helliwell L, Jackson C (2010) Towards evidence based emergency medicine: best BETs from the Manchester Royal Infirmary. BET 1: intranasal fentanyl or diamorphine versus intravenous morphine for analgesia in adults. Emerg Med J 27:715-6

29. Veldhorst-Janssen NM, Fiddelers AA, van der Kuy PH et al (2010) Pharmacokinetics, analgesic effect, and tolerability of a single preprocedural dose of intranasal fentanyl in patients undergoing drain removal after breast reduction or augmentation surgery: a prospective, randomized, double-blind, placebo-controlled study. Clin Ther $32: 1427-36$

30. Panagiotou I, Mystakidou K (2010) Intranasal fentanyl: from pharmacokinetics and bioavailability to current treatment applications. Expert Rev Anticancer Ther 10:1009-21

31. Johnston S, Wilkes GJ, Thompson JA, Ziman M, Brightwell R (2011) Inhaled methoxyflurane and intranasal fentanyl for prehospital management of visceral pain in an Australian ambulance service. Emerg Med J 28:57-63

32. Finn M, Harris D (2010) Intranasal fentanyl for analgesia in the paediatric emergency department. Emerg Med J 27:300-3

33. Chung S, Lim R, Goldman RD (2010) Intranasal fentanyl versus placebo for pain in children during catheterization for voiding cystourethrography. Pediatr Radiol 40:1236-40

34. Holdgate A, Cao A, Lo KM (2010) The implementation of intranasal fentanyl for children in a mixed adult and pediatric emergency department reduces time to analgesic administration. Acad Emerg Med 17:214-7
35. Herd D, Borland M (2009) Intranasal fentanyl paediatric clinical practice guidelines. Emerg Med Australas 21:335

36. Borland ML, Clark LJ, Esson A (2008) Comparative review of the clinical use of intranasal fentanyl versus morphine in a paediatric emergency department. Emerg Med Australas 20:515-20

37. Kress H, Orońska A, Kaczmarek Z et al (2009) Efficacy and tolerability of intranasal fentanyl spray 50 to $200 \mu \mathrm{g}$ for breakthrough pain in patients with cancer: a phase III, multinational, randomized, double-blind, placebo-controlled, crossover trial with a 10-month, openlabel extension treatment period. Clin Ther 31:1177-91

38. Mercadante S, Radbruch L, Davies A et al (2009) A comparison of intranasal fentanyl spray with oral transmucosal fentanyl citrate for the treatment of breakthrough cancer pain: an open-label, randomised, crossover trial. Curr Med Res Opin 25:2805-15

39. Vissers D, Stam W, Nolte T et al (2010) Efficacy of intranasal fentanyl spray versus other opioids for breakthrough pain in cancer. Curr Med Res Opin 26:1037-45

40. Weinstein SM, Messina J, Xie F (2009) Fentanyl buccal tablet for the treatment of breakthrough pain in opioid-tolerant patients with chronic cancer pain: a long-term, open-label safety study. Cancer 115: 2571-9

41. Webster LR, Slevin KA, Narayana A, Earl CQ, Yang R (2013) Fentanyl buccal tablet compared with immediate-release oxycodone for the management of breakthrough pain in opioid-tolerant patients with chronic cancer and noncancer pain: a randomized, double-blind, crossover study followed by a 12 -week open-label phase to evaluate patient outcomes. Pain Med 14:1332-45

42. Rauck R, Tark M, Reyes E et al (2009) Efficacy and long-term tolerability of sublingual fentanyl disintegrating tablet in the treatment of breakthrough cancer pain. Curr Med Res Opin 25:2877-85

43. Radbruck L, Torres LM, Ellershaw JE et al (2012) Long-term tolerability, efficacy and acceptability of fentanyl pectin nasal spray for breakthrough cancer pain. Support Care Cancer 20:565-73

44. Taylor D, Radbruck L, Revnic J, Torres LM, Ellershaw JE, Perelman M (2014) A report on the long-term use of fentanyl pectin nasal spray in patients with recurrent breakthrough pain. J Pain Symptom Manag 47:1001-7

45. Kongsgaard UE, Eeg M, Greisen H (2014) The use of Instanyl in the treatment of breakthrough pain in cancer patients: a 3-month observational, prospective, cohort study. Support Care Cancer 22:1655-62

46. Mercadante S, Prestia G, Adile C, Casuccio A (2014) Intranasal fentanyl versus fentanyl pectin nasal spray for the management of breakthrough cancer pain in doses proportional to basal opioid regimen. J Pain 15:602-7 